\title{
Liga de Neurocirurgia, uma experiência de vinte anos
}

\author{
Neurosurgical League, an experience of twenty years
}

Vinicius Alves Cavalieri' ${ }^{1}$, Eberval Gadelha Figueiredo ${ }^{2}$, Manoel Jacobsen Teixeira ${ }^{3}$

Cavalieri, VA, Figueiredo, EG, Teixeira, MJ. Liga de Neurocirurgia, uma experiência de vinte anos/ Neurological League, na experience of twenty years. Rev Med (São Paulo). 2016 jan.-mar.;95(1):30-32.

RESUMO: A liga de neurocirurgia da Faculdade de Medicina da USP (LNC) é uma liga com 20 anos de história. Atualmente conta com 25 membros distribuídos entre o segundo e quinto ano da graduação. Dentre suas atividades (divididas em obrigatórias e opcionais) temos: aulas teóricas, atividades de enfermarias com discussão de casos clínicos, plantões no pronto-socorro, acompanhamento de procedimentos neurocirúrgicos, dissecções no Serviço de Verificação de Óbito da Capital (SVOC), e atividades de iniciação científica. Durante todo esse período conseguimos disseminar informações sobre a especialidade e consolidar competências nos estudantes de medicina não preenchidas pelo currículo atual. Já produzimos diversos artigos publicados em revistas indexadas nacional e internacionalmente (apresentados em mais de 50 congressos), ajudamos a escrever um livro voltado para alunos de graduação e produzimos diversos cursos de aprimoramento para os alunos.

DESCRITORES: Educação médica; Neurocirurgia/educação; Educação continuada; Educação médica/métodos; Ensino/ métodos; Educação de graduação em medicina; Aprendizagem.
Abstract: The Neurosurgical League of University of São Paulo Medical School is an Academic Interest Group with twenty years of history. At present, it has twenty five members distributed between second and fifth year of undergraduation in medicine. Among its activities (divided into two types: mandatory and optionals) are: lectures, activities in the ward with case discussion, observation of neurosurgical procedures, on call in the neurosurgery emergency, anatomical dissection on the Serviço de Verificação de Óbitos da Capital (SVO), and scientific projects. Throughout this period, we have spread information about the speciality and consolidated competences in the medical students which is not fulfilled by the traditional curriculum. In all these years, the league have produced several published articles in many journals nationally and internationally (presented in more than fifty congresses), have helped to write a book about neurosurgery to undergraduation students and organized continuing education courses.

Keywords: Education, medical; Neurosurgery/education; Education, continuing; Educational, medical/methods; Teaching/ methods; Education, medical, undergraduate; medical; Learning.

1. Graduando de Medicina na Faculdade de Medicina da USP. Email: vinicius.cavalieri@fm.usp.br.

2. Professor Associado do Departamento de Neurologia, Disciplina de Neurocirurgia da Faculdade de Medicina da USP. Email: ebgadelha@yahoo.com.

3. Professor Titular da disciplina de Neurocirurgia da Faculdade de Medicina da USP. Email: manoeljacobsen@gmail.com.

Endereço para correspondência:Vinicius A. Cavalieri. Rua Alves Guimarães, 623 - Apt. 12. São Paulo, SP, Brazil. CEP: 05410-001. Email: vinicius.cavalieri@fm.usp.br. 


\section{INTRODUÇÃO} A formação médica no Brasil tem as suas destoante do restante das outras escolas do mundo sejam as ligas acadêmicas. Elas ganharam forma há dezenas de anos atrás (a primeira de que temos registro na Faculdade de Medicina da USP foi a do Combate a Sífilis e outras DST's, com mais de 90 anos de história) ${ }^{1}$ e se perpetuaram até hoje como uma iniciativa de parte dos acadêmicos para aperfeiçoar a própria educação médica ${ }^{2-4}$.

Formadas inicialmente por um grupo de acadêmicos guiados por um professor, a entidade "liga acadêmica" surgia como uma iniciativa voluntária de combater uma questão de saúde pública. Foi dessa forma que surgiram ligas tão clássicas como a do Combate a Sífilis, a liga do Combate a Febre Reumática, entre outras. Contudo, com o passar dos anos, outros valores e ideias foram se agregando ao conceito de Liga Acadêmica, culminando na atual "trinômia universitária" que deveria ser o objetivo principal de todas as ligas ${ }^{5,6}$.

A trinômia (constituída por ensino, pesquisa e extensão) é, portanto, o principal foco da Liga de Neurocirurgia da Faculdade de Medicina da USP (LNC), atingindo esses objetivos por meio de atividades extracurriculares que objetivam sedimentar e complementar o conhecimento adquirido ao longo da graduação. Nossa liga foi fundada em 1996 com a ajuda e auxílio do Professor Doutor Fernando Campos Gomes Pinto, na época, médico residente do serviço de Neurocirurgia do Hospital das Clínicas. Atualmente, 20 anos depois, a LNC conta com cerca de 15 membros distribuídos entre o terceiro e segundo ano da graduação e com Orientação do Professor Doutor Eberval Gadelha Figueiredo, do Professor Doutor Manoel Jacobsen Teixeira e do Doutor Carlo Emanuel Petitto.

Além disso, no ano de 2000, buscando atender as diretrizes de integração dos diversos profissionais do serviço de saúde, e construir nos membros um espírito de integração e trabalho em equipe, a liga passou a aceitar alunos de outras áreas e especialidades afins. Atualmente trabalhamos em projetos piloto para ampliar essa integração.

\section{ANÁLISE E DISCUSSÃo}

\section{O ingresso e permanência}

Atualmente, a liga é aberta apenas para alunos de graduação da USP das áreas de enfermagem, fisioterapia e fonoaudiologia. O Ingresso se dá por meio de uma prova que cobra conhecimentos básicos de neurocirurgia (como anatomia, fisiologia, exame clínico neurológico) baseados nos temas exibidos em um curso introdutório realizado no começo do ano. São aceitos alunos do $2^{\circ}$ ao $6^{\circ}$ ano da graduação, podendo permanecer até o final do curso de graduação. O número de membros é fixo em 25 alunos. A avaliação dos alunos dá-se, basicamente, por frequência às atividades e interesse.

\section{As atividades}

Cada mês de atividade tem uma temática específica dentro das diversas áreas da Neurocirurgia. Assim, ao longo de um ano o aluno entra em contato com mais de 10 áreas. Nossas atividades são globalmente divididas em dois tipos: as obrigatórias e as optativas.

Dentro das atividades obrigatórias contamos com aulas teóricas sobre um tema específico (ministradas uma ou duas vezes no mês) às quartas-feiras pelos professores ou residentes do departamento e da divisão de neurocirurgia do Hospital das Clínicas (sempre procurando abordar aspectos práticos, demonstrando vias de acesso e manejo clínico do paciente). Além disso, alternadamente com as aulas temos discussão de casos clínicos na enfermaria do Hospital das Clínicas (ilustrando, na prática, ainda mais os casos discutidos na aula teórica).

No Ramo das atividades eletivas são basicamente três opções: dissecções no SVO, acompanhamento de cirurgias e plantões no pronto socorro. Dentre todas, a mais procurada é a dissecção no Serviço de Verificação de Óbitos. Lá dentro, os alunos encontram, toda sexta-feira à noite, um cadáver separado em uma sala especial equipada com microscópio neurocirúrgico e instrumental específico para treinarem os acessos. Sempre acompanhados pelos residentes (que orientam e instruem), os alunos possuem a possibilidade de praticar os diferentes acessos, fundamentando um conhecimento até então puramente teórico. Fora isso, é feito um estudo neuroanatômico, mostrando a disposição real das estruturas cerebrais no momento da cirurgia do ponto de vista do neurocirurgião, ampliando o conhecimento da anatomia cerebral.

Outra opção interessante para os membros é o acompanhamento de neurocirurgias. Dentro do complexo HC são realizados dezenas de neurocirurgias eletivas todos os dias, nos mais diversos institutos. Os membros da liga possuem livre acesso a qualquer uma delas, desde que avisado com antecedência para programação do neurocirurgião (assim o aluno possui a liberdade de acompanhar cirurgias durante seus horários livres). A cada ano procuramos estabelecer um dia preferencial para as visitas ao centro cirúrgico pois, com essa prática, observa-se maior adesão e aproveitamento dessa atividade.

Por fim, oferecemos ainda uma opção de presenciar o cotidiano de um neurocirurgião, isso é feito por meio dos plantões no pronto-socorro. São plantões curtos, geralmente de 4 a 5 horas, no qual os alunos acompanham a rotina de um neurocirurgião durante seu plantão. Essa atividade consagra-se por seu cunho elucidativo da rotina dessa especialidade médica, como também, pela oportunidade de acompanhar pacientes em quadros instáveis. 


\section{A produção científica}

Ao longo de todos esses anos, a liga incentivou os alunos a participarem de atividades científicas. Com mais de 50 apresentações em congressos nacionais e internacionais e diversos artigos publicados em revistas indexadas, buscamos sempre fomentar nos alunos o lado científico, auxiliando, não apenas os que seguirão a carreira neurocirúrgica, mas trabalhando diversas competências exigidas em um bom médico.

Possuímos um dia exclusivo para reuniões científicas periódicas, onde os membros interessados são apresentados a uma iniciação científica e instruídos nas diversas nuances do mundo científico. Nesse dia, o professor orientador discute o andamento dos projetos, analisa conjuntamente com os alunos os artigos científicos, corrige e explica metodologias e delimita novas tarefas para próxima reunião. Concomitantemente os alunos mais velhos, auxiliam aos mais novos dentro do processo, seja explicando a estrutura de um artigo, até mesmo elucidando as diversas formas de se buscar artigos nas bases de dados.

Temos como um grande fruto das atividades científicas da Liga a publicação de um livro sobre neurocirurgia para alunos de graduação. Intitulado "Manual de Iniciação em Neurocirurgia" de autoria do Professor

\section{REFERÊNCIAS}

1. Burjato Junior D. Academic League history to combat syphilis and evolution of syphilis in the city of São Paulo (1920-1995). School of Medicine, University of São Paulo; 1999.

2. De Souza Aguiar LF, Méndez CKI, Gonçalves EG, Gomes FP, Gesser Cardoso FR, Nozaki GY, et al. Medical surgery leagues. Aesthetic Plast Surg. 2013;37(2):485-8. doi: 10.1007/ s00266-012-0043-7.

3. Silva JBG da, Saidah R, Megid CBC, Ramos NA. Teaching acupuncture to medical students: the experience of Rio Preto Medical School (FAMERP), Brazil. Acupunct Med. 2013;31(3):305-8. doi: 10.1136/acupmed-2013-010370.
Doutor Fernando Campos Gomes Pinto, o livro é, até hoje, é muito usado e vendido nas livrarias do Brasil por curiosos que pretendem aprofundar seus conhecimentos sobre essa área da medicina.

Além disso, promovemos cursos para a comunidade acadêmica, como o Curso de Neuroanatomia Tridimensional. No qual, usando de tecnologia 3D, busca-se ilustrar de maneira mais próxima e dinâmica conhecimentos anatômicos básicos que todos os graduando deveriam possuir.

\section{CONCLUSÃO}

Considerando o tempo de existência e o numero de acadêmicos que já frequentaram a liga, sua produção científica e as suas atividades, concluímos que ela atua de forma importante como instrumento de desenvolvimento da educação médica fechando "lacunas" do currículo e fixando conceitos básicos de neuroanatomia e afecções comuns do sistema nervoso. E, por fim, age como fomentadora do processo científico, promovendo o desenvolvimento de ferramentas para constante atualização médica, desenvolvimento de conhecimento novo.

4. Ramalho AS, Silva FD, Kronemberger TB, Pose RA, Torres MLA, Carmona MJC, et al. Anesthesiology teaching during undergraduation through an academic league: what is the impact in students' learning? Rev Bras Anestesiol. 2012;62(1):63-73. doi: 10.1016/S0034-7094(12)70104-6.

5. Pego-Fernandes PM, Mariani AW. Medical teaching beyond graduation: undergraduate study groups. Sao Paulo Med J. 2010;128(5):257-8. doi: 10.1590/S1516-31802010000500002.

6. Torres AR, Oliveira GM de, Yamamoto FM, Lima MCP. Academic Leagues and medical education: contributions and challenges. Interface - Comun Saúde Educ. 2008;12(27):71320. doi: 10.1590/S1414-32832008000400003. 